Sweat distribution and perceived wetness across the human foot: the effect of shoes and exercise intensity

West, Anna.M ${ }^{\mathrm{a}}$. Tarrier, James ${ }^{\mathrm{b}}$. Hodder, Simon ${ }^{\mathrm{a}}$. and Havenith, George ${ }^{\mathrm{a}}{ }^{*}$.

${ }^{a}$ Environmental Ergonomics Research Centre, Loughborough Design School,

Loughborough University, Loughborough, UK; ${ }^{b}$ adidas FUTURE, adidas AG-World of

Sports, Herzogenaurach, Germany

*Correspondence

Professor George Havenith

Environmental Ergonomics Research Centre (James France Building),

Loughborough Design School,

Loughborough University,

Loughborough,

LE11 3TU, UK

E: g.havenith@lboro.ac.uk

ORCID: 0000-0001-6223-4265 


\section{Sweat distribution and perceived wetness across the human foot: the effect of shoes and exercise intensity}

This study investigates foot sweat distribution with and without shoes and the relationship between foot sweat distribution and perceived wetness to enhance guidance for footwear design. Fourteen females performed low-intensity running with nude feet and low- and high-intensity running with shoes $\left(55 \% \mathrm{VO}_{2 \max }\right.$ and $75 \% \mathrm{VO}_{2 \max }$, respectively) on separate occasions. Right foot sweat rates were measured at 14 regions using absorbent material applied during the last 5 minutes of each work intensity. Perceptual responses were recorded for the body, foot and four foot regions. Foot sweat production was $22 \%$ greater nude $(\mathrm{p}<0.001)$ and with shoes did not increase with exercise intensity $(\mathrm{p}=0.14)$. Highest sweat rates were observed at the medial ankle and dorsal regions; lowest sweat rates at the toes. Perceptions of wetness and foot discomfort did not correspond with regions of high sweat production or low skin temperature but rather seemed dominated by tactile interactions caused by foot movement within the shoe.

Keywords: sweat mapping; regional sweating; feet; exercise; footwear; comfort

Practitioner summary: This study provides a detailed view of foot sweat distribution for female runners with and without shoes, providing important guidance for sock and footwear design. Importantly, perceptions of wetness and foot discomfort did not correspond with areas of high sweat production. Instead tactile interactions between the foot, sock/shoe play an important role. 


\section{Introduction}

The study of sweat distribution for different body regions (sweat-mapping) has gained attention in recent years (Havenith et al. 2008; Machado-Moreira, Smith, et al. 2008; Machado-Moreira, Caldwell, et al. 2008; Smith and Havenith 2011, 2012, 2019) after early work mainly focused on the global sweat response. Through a whole body sweat-mapping approach a more detailed picture of the variations in sweat rate across the body has been identified. Highest sweat rates have been observed on the central (upper and mid) and lower back (Smith and Havenith 2011, 2012; Machado-Moreira, Smith, et al. 2008). Lowest values have been observed at the extremities, particularly at the hands and feet (Smith and Havenith 2011; Machado-Moreira, Caldwell, et al. 2008; Smith et al. 2013). The benefit of such an approach has led to functional design improvements to protective clothing and sportswear.

The feet account for $7 \%$ of body surface area, yet foot sweat loss only accounts for 3-4\% of whole body sweat loss (Weiner 1945; Smith and Havenith 2011; Smith et al. 2013; Taylor, Caldwell, and Mekjavic 2006). Although foot sweat production is relatively small, the feet are often contained within shoes which impair the dissipation of heat and evaporation of sweat from its surface resulting in high temperatures and high humidity within the shoe (Arezes et al. 2013; Irzmanska, Dutkiewicz, and Irzmanski 2013; West et al. 2019). The combination of high temperatures and humidity contribute to perceptions of foot discomfort (West et al. 2019) and encourage the growth of microorganisms which can lead to odour development and to poor foot health, including tinea pedis (Auger et al. 1993) and blister formation (Sulzberger et al. 1966). Knowledge regarding foot sweat rates and distribution are therefore important in the design of footwear, especially as footwear manufacturers seek to optimise shoes to improve foot thermal comfort and foot health.

Depending on the sweat measurement technique (ventilated sweat capsules vs technical absorbents) and experimental protocol implemented (one-legged incremental cycling, with sweat rates being measured from the stationary nude foot [Taylor et al. 2006] vs low and high intensity running with footwear [shod] [Smith et al. 2013]), each foot has been estimated to secrete between 23 and $27 \mathrm{~g} . \mathrm{hr}^{-1}$ respectively. To add to this, investigations of foot sweat rates consistently report a non-uniform distribution of sweat across the foot surface with sweat rates being greatest from the dorsal surface compared to the plantar surface ( $\sim 70 \%$ versus $\sim 30 \%$ respectively) (Fogarty et al. 2007; Smith et al. 2013; Taylor, Caldwell, and Mekjavic 2006). 
Comparisons between investigations of foot sweat rates (Taylor, Caldwell, and Mekjavic 2006) report that when the passive foot is nude, foot sweat rates progressively increase with both increased exercise load performed by the other foot and with the level of heat strain. However, when footwear is worn (Fogarty et al. 2007; Smith et al. 2013), despite an increased exercise load, foot sweat rates are suppressed. This observation has been suggested to be the effect of hidromeiosis (skin swelling due to excessive hydration, closing the sweat ducts) (Candas, Libert, and Vogt 1980; Kerslake 1972). As these observations on shod and nude feet are for test protocols differing in environmental conditions, heating techniques and exercise modalities, no final conclusion on this issue can be drawn. Hence, in the present study regional foot sweat distribution was investigated using the same protocol at two exercise intensities when shod and at one exercise intensity when the foot was nude. Sweat distribution for 14 foot regions were assessed providing the most detailed overview of foot sweat rates to date.

Assessment of regional sweat distribution across the foot is also important in our understanding of foot thermal comfort. Skin wettedness has been repeatedly shown to play a significant role in the onset of thermal and clothing discomfort (Fukazawa and Havenith 2009; Raccuglia et al. 2017, 2018) and more recently has been shown to play a role in the onset of foot discomfort (West et al. 2019). Considering we are not provided with specific receptors for the sensation of wetness or humidity, these sensations are suggested to be the result of thermal (heat transfer) and tactile (skin friction and stickiness) inputs produced from the interaction between the skin, moisture and clothing (Bergmann Tiest et al. 2012; Davide Filingeri and Havenith 2015). These inputs are particularly high at the foot due to unfavourable changes to shoe microclimate and the tactile inputs generated due to foot-sock-shoe interactions (West et al. 2019). Surprisingly, the perception of wetness in relation to foot sweat distribution has not been investigated. In the present paper, these relations are explored specifically for the feet and by studying shod and nude feet a large difference in thermal and tactile inputs is created.

Moreover, the majority of research available and five of the eight papers discussed above focuses on males rather than females (Smith and Havenith 2011, 2019; MachadoMoreira, Smith, et al. 2008; Machado-Moreira, Caldwell, et al. 2008; Taylor, Caldwell, and Mekjavic 2006). The first study to provide a comparison of regional sweat rates across the whole body between males and females was published by Smith and Havenith (2012). Differences in sweat distribution were observed between sexes, although high and low sweat regions were similar. Further investigations of sweating in females are 
limited to the upper body (Havenith et al. 2008). Given the paucity of female data, it was decided to investigate foot sweat rates in females.

As is evident from the above, foot sweat distribution has been studied yet many questions remain unanswered. Therefore, the aims of this research were to investigate (1) how foot sweat distribution varies with exercise intensity and when the foot is nude versus shod and (2) how the perception of wetness relates to foot sweat distribution. The applications of this research include the advancement of thermal foot manikin design (Kuklane and Holmer 1998) and the design of footwear.

\section{Materials and methods}

\subsection{Participants}

Fourteen trained female runners (age: $23 \pm 3.6 \mathrm{yrs}$; height: $166.4 \pm 5.3 \mathrm{~cm}$; body mass: $56.7 \pm 8.2 \mathrm{~kg} ; \mathrm{VO}_{2 \max } 52.1 \pm 4.9 \mathrm{ml} \cdot \mathrm{kg}^{-1} \cdot \mathrm{min}^{-1}$ ) participated in foot sweat mapping trials. These were identified as individuals running $10 \mathrm{~km}$ in under 50 minutes. Test procedures were outlined in a written information sheet and were explained to participants before obtaining written informed consent and completing a health screen questionnaire. Experimental procedures were approved by Loughborough University Ethical Committee.

\subsection{Pre-test session}

Participants were required to attend the laboratory for a pre-test session involving anthropometric measurements of height, body mass and body composition. Anthropometric measurements of the right foot were taken to determine absorbent pad dimensions for sweat rate analysis. All participants were right foot dominant. Participants were introduced to subjective scales and instructed on their use.

Maximal oxygen consumption $\left(\mathrm{VO}_{2 \max }\right)$ was estimated from a sub-maximal fitness test performed on a motorised treadmill (PPS 55sport-I, Woodway GmbH, Weil am Rhein, Germany) (American College of Sports Medicine 2017). The sub-maximal test comprised of four exercise intensities each lasting five minutes. The test was designed to achieve $85 \%$ of heart rate (HR) maximum after which the test was stopped. Estimation of $\mathrm{VO}_{2 \max }$ was calculated based upon the linear relationship between $\mathrm{VO}_{2}$ and $\mathrm{HR}$. 


\subsection{Experimental preparation}

\subsubsection{Sweat pad preparation and application}

Regional foot sweat rates were determined by applying absorbent material (Product 2164 - Laminated Airlaid Tech Absorbents, Grimsby, UK) directly to the skin of the right foot only for a short, predefined period of time. Although local sweat rates have more commonly been assessed using the ventilated capsule method, the technical absorbent method allows for local sweat rate measurements to be taken when wearing shoes and while the foot is active. The technical absorbent method has been shown to be highly correlated with local sweat rates measured using the ventilated capsule method, irrespective of sample surface area and body region (Morris et al. 2013).

Based upon anthropometric measurements taken during the pre-test, three sets of absorbent pads were produced for each participant, measuring sweat rate from 14 foot regions (Figure 1). For fast application to the measured foot, absorbent pads for the plantar and dorsal foot surface were kept together as one piece. To separate the regions and to prevent the migration of sweat during collection, a width of $0.5 \mathrm{~cm}$ of absorbent material was cut away from the plastic backing along the borders of the marked regions. A $100 \%$ cotton toe sock (Bonamart, UK) was used to collect sweat for the toes. Latex covers were placed over each toe to prevent sweat evaporation in the measurement period.

\section{[Figure 1 near here]}

\subsection{Experimental protocol}

Participants were advised to consume $20 \mathrm{~mL} \cdot \mathrm{kg}^{-1}$ of water $2 \mathrm{hrs}$ prior to testing to maintain a euhydrated state and were allowed to drink water freely during experimental sessions. Participants were advised against alcohol intake $24 \mathrm{hrs}$ before testing and against the consumption of food and caffeine within $2 \mathrm{hrs}$ of experimentation. Participants performed trials at the same time of day to minimise the effect of circadian rhythm. Female menstrual cycle phase was not controlled for during experimental sessions. Instead, menstrual cycle phase for each participant was noted with a range of phases being tested during the experiment, thus providing a representative sample of menses states in the results. 
Experimental sessions were conducted in a climate-controlled chamber at $25^{\circ} \mathrm{C}$, $50 \% \mathrm{RH}, 2 \mathrm{~m} . \mathrm{s}^{-1}$ frontal air velocity. On arrival to the laboratory, participants were provided with standardised clothing (shorts and t-shirt). Participants were weighed prior to each run and immediately after each trial. Infrared images of the dorsal and plantar surfaces of the feet were taken prior to testing and before and after each pad application. Core body temperature was monitored continuously and recorded at $60 \mathrm{~s}$ intervals (Squirrel 2020 data logger) using a thermistor self-inserted $10 \mathrm{~cm}$ beyond the anal sphincter (Grant Instruments, Cambridge, UK). Heart rate was monitored throughout the experimental protocol and recorded at $60 \mathrm{~s}$ intervals (Polar RS400sd, Polar Electro Oy,Kempele, Finland). Skin temperature was measured at four sites: chest, upper arm, thigh and calf using iButton wireless temperature loggers (Maxim, San Jose, USA) attached to the skin with $3 \mathrm{M}^{\mathrm{TM}}$ Transpore ${ }^{\mathrm{TM}}$ surgical tape (3M United Kingdom PLC). The weighted skin temperature of four sites was calculated according to Ramanathan (1964).

Subjective perception of thermal sensation, wetness perception, stickiness and thermal comfort were assessed using ordinal scales (Figure 2a-d). Scales were designed in line with instructions from ISO 10551 (2001) with the sensitivity, accuracy, ease of use and descriptors chosen for each individual scale carefully considered.

Scales were used to gain subjective information including thermal sensation and thermal comfort for the whole body and thermal sensation, wetness perception, stickiness and thermal comfort for the foot and four foot regions (dorsal, toes, sole and heel: Figure 2e). Subjective information was obtained for the right foot only. Participants were prompted every 5 mins to provide subjective votes which took between 1-2 mins. Subjective information was not recorded during pad application.

\section{[Figure 2 near here]}

Participants were required to attend two experimental sessions completing either 60 minutes or 35 minutes of treadmill running with a $1 \%$ incline. The 60 minute protocol was performed shod (socks and running shoes) and consisted of 35 minutes low intensity running (shod-I1: 55\% $\mathrm{VO}_{2 \max }$; HR 125-135 beats $\mathrm{min}^{-1}$ ) followed by 25 minutes of higher intensity running (shod-I2: 75\% $\mathrm{VO}_{2 \max }$; HR 150-160 beats $\min ^{-1}$ ). The 35 minute protocol was performed bare-foot (nude), running at a low exercise intensity (nude-I1: 
$55 \% \mathrm{VO}_{2 \max }$; HR $125-135$ beats $\min ^{-1}$ ). Higher intensity running was not performed nude due to high discomfort reported by participants and blister formation during pilot testing.

Socks used were adidas performance ankle socks ( $94 \%$ cotton, $3 \%$ polyamide, $3 \%$ elastane, terry jersey). Running shoes (adidas adizero adios 3.0) had a heat resistance of $0.09 \mathrm{~m}^{2} . \mathrm{K}^{-\mathrm{W}^{-1}}$ and a vapour resistance of $16.7 \mathrm{~m}^{2} . \mathrm{Pa} . \mathrm{W}^{-1}$ determined according to ISO 15831 (2004) on a 12 zone sweating thermal foot manikin (THERMETRICS, Measurement Technology Northwest, Seattle, USA). These socks and shoes were chosen to show the impact of a footwear ensemble on the foot sweat responses whilst at the same time having minimal impact on that response. For example, the shoes were minimal in design features and had a relatively uniform level of insulation across the shoe upper.

During the 60 minute protocol, sweat pads were applied first following 30 minutes of low intensity running for 5 minutes and again for 5 minutes after 20 minutes of high intensity running. Participants ceased running, removed socks and shoes and towel dried their feet. Infrared pictures of the dorsal and plantar foot surface were recorded to evaluate local foot skin temperature (T620, FLIR Systems Ltd, West Malling, Kent, UK). Absorbent pads and the sweat collection toe socks were taken out of their plastic bags and applied to the skin. Shoes were donned and participants continued their run for the 5 minute sweat collection phase. At the conclusion of this period, the absorbent pads and toe socks were removed quickly and sealed in their air tight bags, the skin dried and infrared images taken. Participants dressed without pads to continue the test. In the nude condition, the application of absorbent pads was as above but did not require removing footwear.

Given the time needed for the application of the pads, not the whole application period consisted of exercise, possibly resulting in a slight under estimation of sweat rates for the workload. However, core body temperature and skin temperature (drivers of sweating) were not greatly affected by the short interruption and so this effect is assumed to be small and would not affect the comparison of the regions.

\subsection{Post-experimental protocol}

\subsubsection{Weighing of absorbent pads and cotton socks}

The individual sections of the plantar and dorsal absorbent pads and individual toes of the cotton socks could not be weighed prior to testing. These specific areas of the absorbent pads and socks were cut up immediately following sweat collection and placed 
in individually labelled airtight bags. The wet weight of each individual section was weighed using electronic scales with a resolution of $0.001 \mathrm{~g}$ (FX-500i. A\&D Company Ltd, Oxfordshire, UK) recorded and then removed from its bag. Each section was placed in a room at $25^{\circ} \mathrm{C}, 40 \% \mathrm{RH}$ for $24 \mathrm{hrs}$ to allow the material to dry. The sections of absorbent pad and cotton sock were re-weighed inside their individual labelled airtight bags to establish the dry weight. Absorbent pads used on the ankles were weighed pre and post application.

\subsection{Analysis}

Gross sweat loss (GSL) was calculated based on the mass change of each participant from the start to end of each running bout, adjusted for fluid consumption and corrected for respiratory and metabolic mass losses.

Regional foot sweat rates were calculated in grams per metre squared per hour based upon the change in pad mass, surface area and the duration of application to the skin (Havenith et al. 2008; Smith and Havenith 2011):

$$
\text { Foot sweat rate }=\frac{\left[\frac{w_{w}-w_{d}}{S A}\right]}{t} \cdot 3600
$$

Where;

$w_{w}=$ wet weight of individual pad/cotton $(g)$

$w_{d}=$ dry weight of individual pad/cotton $(g)$

$S A=$ surface area of individual pad $/ \operatorname{cotton}\left(\mathrm{m}^{2}\right)$

$t=$ duration of pad/cotton application $(s)$

\subsection{Statistical analysis}

To investigate regional sweat distribution differences across the foot in relation to condition and intensity, two one-way repeated measured ANOVA's were performed. Regional skin temperature data were analysed using the same method as for regional sweat rate data. Data which deviated from normal distribution was assessed using Wilcoxon signed rank tests. To investigate the subjective perception of foot sweat rate across foot zones in relation to condition and intensity Friedman tests were performed. When significant effects were observed, post hoc analysis was conducted with a Wilcoxon signed rank test. Pearson correlation coefficient analyses were performed to 
study the relationships between foot sweat rate and foot skin temperatures as well as foot perceptual responses and foot sweat rate. Statistical analysis was performed using IBM SPSS Statistics 24 (IBM, USA) and reported as means \pm standard deviation (SD). The level of significance was set at $p<0.05$. 


\section{Results}

Mean $( \pm \mathrm{SD})$ environmental conditions for the experimental trials were $24.9 \pm$ $0.2^{\circ} \mathrm{C}, 49.5 \pm 0.7 \% \mathrm{RH}$.

\subsection{Core temperature and mean skin temperature}

No difference in core temperature $\left(37.7 \pm 0.3^{\circ} \mathrm{C}\right.$ vs $\left.37.8 \pm 0.2^{\circ} \mathrm{C}, p=0.18\right)$ or mean skin temperature $\left(31.2 \pm 0.8^{\circ} \mathrm{C}\right.$ vs $\left.31.3 \pm 0.9^{\circ} \mathrm{C}, p=0.19\right)$ were present between nude-I1 and shod- I1, respectively. With exercise intensity (shod-I1 to shod-I2), core temperature significantly increased from $37.8 \pm 0.2^{\circ} \mathrm{C}$ to $38.2 \pm 0.3^{\circ} \mathrm{C}(p<0.001)$, as did mean skin temperature $\left(31.3 \pm 0.9^{\circ} \mathrm{C}\right.$ to $\left.32.0 \pm 1.2^{\circ} \mathrm{C}, p=0.001\right)$.

\subsection{Gross sweat loss}

No significant difference was observed in GSL between nude-I1 (277 \pm 66 g.m ${ }^{-}$ $\left.{ }^{2} \cdot \mathrm{h}^{-1}\right)$ and shod-I1 $\left(275 \pm 73 \mathrm{~g} \cdot \mathrm{m}^{-2} \cdot \mathrm{h}^{-1}\right)$. GSL increased significantly from $275 \pm 73$ to 360 $\pm 63 \mathrm{~g} \cdot \mathrm{m}^{-2} \cdot \mathrm{h}^{-1}$ with exercise intensity $(p<0.001)$.

\subsection{Foot sweat rate}

Foot sweat rate was significantly greater $(p<0.001)$ when nude-I1 $(262.9 \pm 75.8$ g. $\left.\mathrm{m}^{-2} \cdot \mathrm{h}^{-1}\right)$ compared to shod-I1 $\left(212.0 \pm 73.5 \mathrm{~g} \cdot \mathrm{m}^{-2} \cdot \mathrm{h}^{-1}\right)$. Foot sweat rate did not increase $(p=0.14)$ with shod-I2 $\left(222.3 \pm 72.3 \mathrm{~g} \cdot \mathrm{m}^{-2} \cdot \mathrm{h}^{-1}\right)$.

\subsection{Regional foot sweat rates}

Regional foot sweat distribution for nude-I1, shod-I1 and shod-I2 are illustrated in Figure 3. The low sweat rates of the toes compared to the dorsal and plantar regions are clearly presented. Little change in sweat distribution is observed between condition and intensity. A comparison of sweat rates by region for nude-I1 and shod-I1 and for shod-I1 to shod-I 2 is presented in Table 1 with descriptive statistics for all regions tested.

[Figure 3 near here]

[Table 1 near here] 


\subsection{Foot skin temperature}

Mean foot skin temperature significantly increased from baseline to pre-pad application for nude-I1 (baseline: $24.8 \pm 1.2^{\circ} \mathrm{C}$, pre pad: $32.1 \pm 1.0^{\circ} \mathrm{C}, p<0.001$ ) and shod-I1 (baseline: $24.6 \pm 1.5^{\circ} \mathrm{C}$, pre pad: $33.9 \pm 0.5^{\circ} \mathrm{C}, p<0.001$ ), reflecting low baseline values. Mean foot skin temperature significantly increased with exercise intensity (shodI1 post pad: $33.3 \pm 0.7^{\circ} \mathrm{C}$, shod-I 2 pre pad: $\left.34.3 \pm 0.6^{\circ} \mathrm{C}, p<0.001\right)$.

Mean foot skin temperature was $1.8^{\circ} \mathrm{C}$ higher pre-pad application $(p<0.001)$ and $1.0^{\circ} \mathrm{C}$ higher post-pad application $(p=0.01)$ for shod-I1 compared to nude-I1. Mean foot skin temperature was $0.4^{\circ} \mathrm{C}$ higher pre- and post-pad application for shod-I 2 compared to shod-I1 $(p<0.01)$.

To assess the impact of the sweat measurement procedure on skin temperature, the change in foot skin temperature between pre- and post-pad application was reviewed. During pad application, mean foot skin temperature increased by $0.2^{\circ} \mathrm{C}$ during nude-I1 $(p=0.15)$ and decreased by $0.6^{\circ} \mathrm{C}$ during shod-I1 and shod-I $2(p<0.001)$. When assessed by foot regions, a significant increase in foot skin temperature was only observed at the dorsal region pre- to post-pad application during nude-I1 $\left(+0.6^{\circ} \mathrm{C}, p=0.001\right)$. Foot skin temperature across remaining foot regions significantly decreased pre-to post-pad application across all conditions $\left(<-1.0^{\circ} \mathrm{C}, p<0.05\right)$.

\subsection{Relation between foot skin temperature and foot sweat rate}

No significant correlations were observed between mean foot skin temperature pre- or post-pad application and mean foot sweat rate for nude-I1, shod-I1 or shod-I2 ( $\mathrm{r}^{2}$ $<0.18$ ). Significant correlations were also not observed between regional foot skin temperature and regional foot sweat rates for all conditions.

\subsection{Foot perceptual responses}

Thermal sensation, wetness perception and stickiness were greater during shodI1 compared to nude-I1 (Figure $4 ; p<0.05$ ). Although general trends indicated a greater level of thermal discomfort during shod-I1, this did not reach significance $(p=0.20)$ (Figure 4d). With an increase in exercise intensity (shod-I1 to shod-I2), thermal sensation, wetness perception and stickiness increased, resulting in greater thermal discomfort Figure $4(; p<0.001)$. 


\subsection{Relation between foot perceptual responses and foot sweat rate}

No significant correlations were observed between foot perceptual responses and foot sweat rate for nude-I1, shod-I1 or shod-I2 $\left(\mathrm{r}^{2}<0.28\right)$. Wetness perception, stickiness and thermal comfort was therefore not associated with the amount of sweat produced.

[Figure 4 near here]

\subsection{Regional foot perceptual responses}

Mean perceptual votes taken prior to pad application for four foot regions (dorsal, toes, heel and sole) during nude-I1 and shod-I1 (minute 30) and for shod-I2 (minute 55) are shown in Figure 5. Across all conditions, thermal sensation, wetness perception, stickiness and thermal discomfort were greatest at the toes and heel. In most cases, these regions were significantly different from dorsal and sole regions $(p<0.05)$.

\subsection{Relation between regional foot perceptual responses and regional foot sweat rates}

Weak and mostly inconsistent positive and negative correlations were observed between regional foot perceptual responses and regional foot sweat rates. Foot regions of high sweat production were not associated with greater perceptions of wetness, stickiness or thermal discomfort.

[Figure 5 near here] 


\section{Discussion}

The primary aim of this study was to investigate regional foot sweat distribution when the foot was nude and when the foot was shod at two different exercise intensities in trained female runners. A secondary aim of the study was to assess the relationship between perceived wetness and sweat distribution across the human foot. The data have illustrated large inter-individual variation in foot sweat rates with a substantial regional variation. Sweat distribution across the foot surface was similar for all studied conditions and exercise intensities. Importantly regions of high sweat production are not perceived as being regions of high wetness perception or thermal discomfort pointing to an important role of tactile stimulation in the perception of wetness and discomfort.

\subsection{Foot sweat loss}

Single foot sweat loss was $22 \%$ greater for nude-I1 compared to shod-I1 (12.6 \pm 3.7 g.hr ${ }^{-1}$ and $10.3 \pm 3.6$ g.hr- ${ }^{-1}, p<0.001$, respectively) despite similar core temperatures, mean skin temperatures, GSL and higher local skin temperatures in the latter. In line with previous investigations of foot sweat rates (Fogarty et al. 2007; Smith et al. 2013), this observation in the shod condition together with the lack of increase in sweat rate from shod-I1 to I2 (10.3 $\pm 3.6 \mathrm{~g} \cdot \mathrm{hr}^{-1}$ and $10.8 \pm 3.5 \mathrm{~g} \cdot \mathrm{hr}^{-1}$ and, $p=0.14$, respectively) may be indicative of hidromeiosis. In hot humid environments, decreases in sweat rate are dependent on evaporative conditions. If maximum evaporative capacity is low due to high in-shoe microclimate water vapour pressure, hidromeiosis will be greater, while nonexistent if all secreted sweat is evaporated (Candas, Libert, and Vogt 1980; Kerslake 1972). In the current study during nude-I1, with a substantially greater maximum evaporative capacity, hidromeiosis would have been unlikely. In contrast, it could be expected that when shod, changes to the shoe microclimate in terms of increased in-shoe temperature and in-shoe relative and absolute humidity (West et al. 2019) would result in high levels of skin wettedness causing the observed reduction in foot sweat rate.

Previous data for foot sweat loss estimate each foot to secrete $27.6 \mathrm{~g}^{\mathrm{hr} \mathrm{h}^{-1}}$ for males when measured using sweat capsules and between 23 g.hr-1 for low intensity activity and 32 g.hr ${ }^{-1}$ for higher activity for male and females when using the technical absorbent method (Smith et al. 2013). With low and high intensity uphill walking for males, Fogarty et al. (2007) reported foot sweat rates to be 15-29 g.hr-1. Whole foot sweat loss in the current study (nude-I1: $12.6 \pm 3.7$ g.hr-1 ${ }^{-1}$, shod-I1: $10.3 \pm 3.6$ g.hr-1 ${ }^{-1}$, shod-I2: $10.8 \pm 3.5$ 
g.hr ${ }^{-1}$ ) therefore appears to be lower than previously reported (Smith and Havenith 2012; Smith et al. 2013). Subsequent analysis of Smith and Havenith's (2012) and Smith et al's. (2013) whole foot sweat rate data indicates that although the authors report whole foot sweat rate per foot, sweat rate has actually been calculated and reported for both feet combined. With this being considered, the sweat rate data reported in the current study is in line with previous research and shows good agreement with the female sweat rate data reported by Smith et al. (2013).

Furthermore, the data in the current study illustrated large variation in whole foot sweat rate between individuals with approximately a factor 4 and a factor 6 difference between the lowest and highest sweat rate for the nude foot and shod foot respectively. Large regional variations in whole body and foot sweat rates between individuals have previously been reported (Smith and Havenith 2011, 2012; Smith et al. 2013).

\subsection{Regional foot sweat loss}

This study demonstrated significant regional variation across the foot surface with foot sweat distribution patterns being similar regardless of condition or exercise intensity. Highest sweat rates were observed from the medial ankle, medial dorsal and central dorsal for shod-I1 and shod-I2 and from the medial dorsal, medial ankle and heel for nude-I1. Lowest sweat rates were always observed at the toes. The medial ankle and dorsal region have previously been identified as areas of high sweat production on the foot surface (Taylor, Caldwell, and Mekjavic 2006; Smith and Havenith 2011; Smith et al. 2013; Fogarty et al. 2007). The observed variations in sweat rate across the foot surface cannot be explained by sweat gland densities. Sweat gland densities have been reported to be highest on the plantar surface (460 glands. $\mathrm{cm}^{-2}$ ) with values ranging from 302 to 620 glands. $\mathrm{cm}^{-2}$. Lower densities have been found on the dorsal surface (175 glands. $\mathrm{cm}^{-2}$ ) ranging from 144-250 glands.cm ${ }^{-2}$ (Szabo 1967; Hwang and Baik 1997). Therefore although $77 \%$ of foot sweat glands are located on the plantar surface they only account for $30 \%$ of foot sweat production. This discrepancy between sweat gland density and sweat production was also observed for the body as a whole by Smith and Havenith (2011). The role of active sweat glands, output per gland and sweat gland sensitivity must therefore be considered (Smith and Havenith 2011).

Comparisons between foot regions in the different conditions indicated greater sweat rates for nude-I1 compared to shod-I1 at the lateral dorsal, heel and outer. Decreased sweat rates from shod-I1 to shod-I2 were observed for the arch, ball and outer 
foot regions. It could be expected that the effect of hidromeiosis would be greater on the plantar surface given the higher moisture accumulation. West et al. (2019) reported the heel and sole to be areas of high in-shoe temperature and in-shoe humidity compared to the dorsal and toe regions. Consequently, a reduced evaporative capacity and therefore high levels of skin wettedness may have resulted in lower foot sweat rates at these regions for shod-I1 compared to nude-I1 and a decrease in sweat rate at these regions for shodI2. In addition, the plantar foot regions experience high pressure during running which in a limited number of studies has been demonstrated to suppress sweat rate (Kuno 1956; Ferres 1960).

\subsection{Foot skin temperature}

Foot skin temperature was higher for the whole foot and by foot regions for shodI1 compared to nude-I1 and for shod-I2 compared to shod-I1. Shoes act as a barrier to heat and moisture transfer, thus resulting in higher foot skin temperatures (Irzmanska, Dutkiewicz, and Irzmanski 2013; West et al. 2019).

Local skin temperature, alongside core and mean skin temperature are suggested to modulate sweat rate (Nadel, Bullard, and Stolwijk 1971). However, foot skin temperature was not effective in explaining differences in foot sweat rates for nude-I1, shod-I1 or shod-I2 in the present data. In support, Smith and Havenith (2011) also did not observe a relationship between regional sweat rate and regional skin temperatures across the whole body. This could be due to skin temperatures remaining high (above $31^{\circ} \mathrm{C}$ in the current study) with little variation between regions. Models of sweat rate as a function of changes to core and skin temperature are based upon much larger variations in skin temperatures (Nadel, Bullard, and Stolwijk 1971) than observed in the current study or in Smith and Havenith (2011).

\subsection{Foot perceptual responses}

Higher thermal warmth sensations were experienced for shod-I1 compared to nude-I1 and with increased exercise intensity. This is in line with observed increases in foot skin temperature. Foot skin temperature elevations have previously been reported to be well perceived during running (Arezes et al. 2013; West et al. 2019). In the present study, the perception of foot wetness however was not associated with foot sweat production. Despite lower foot sweat rate during shod-I1 $\left(10.3 \pm 3.6\right.$ g.hr $\left.{ }^{-1}\right)$ compared to nude-I1 $\left(12.6 \pm 3.7\right.$ g.hr $\left.{ }^{-1}\right)$, and no increase in foot sweat rate with shod-I2 (10.8 \pm 3.5 
g.hr $\left.{ }^{-1}\right)$, participants experienced greater sensations of wetness and stickiness for shod-I1 compared to nude-I1 and with increased exercise intensity, resulting in higher local thermal discomfort.

According to Filingeri et al. (2014) and Raccuglia et al. (2018) wetness is perceived through the integration of thermal (heat transfer) and tactile (friction and stickiness) inputs occurring at the skin. When thermal cues provide insufficient sensory inputs, individuals seem to rely upon tactile inputs (i.e. stickiness resulting from the adhesion of a wet material to the skin) for the sensation of wetness (Bergmann Tiest et al. 2012; Raccuglia et al. 2017). In the present study, foot skin temperatures elicited by condition and intensity were in a high and narrow range $\left(\sim 31^{\circ} \mathrm{C}\right)$. Thus, in the absence of a variation in the temperature stimulus, the increased sensation of wetness in shod-I1 compared to nude-I1 and with increased exercise intensity must be attributed to the increased magnitude of stimulation of cutaneous mechanoreceptors from the mechanical and pressure related sensations generated between the foot, sock and shoe.

\subsection{Regional perceptual responses}

The results from the current study show that foot regions associated with high levels of perceived wetness are not the regions with the highest rates of sweat production. Instead, the heel and toes, areas of low sweat production, are perceived as being the wettest, stickiest and most uncomfortable foot regions regardless of condition or exercise intensity. As mechanical and pressure related sensations could be expected to play an important role in the ability to sense wetness at the foot through tactile perception, it is perhaps not surprising that these regions are experienced as having high wetness. During gait, the heel experiences high contact loads (Shimazaki and Murata 2015) with the toes performing a grabbing action in order to leave the ground and thrust the body forward upon toe-off (Dugan and Bhat 2005). In addition, it could be expected that tactile cues are produced through toe movement not only against the sock and shoe but also between the toes themselves. Thus tactile cues generated between the foot, sock and shoe are strong predictors for wetness sensation at the foot, a phenomenon observed earlier for textile-skin interactions at the torso (Raccuglia et al. 2018, 2017). 


\section{Conclusions}

The present study provides the most detailed view of regional sweat distribution to date for trained female runners when feet are shod and nude. Highest sweat rates were observed at the medial ankle and dorsal regions. Lowest sweat rates were observed at the toes. Foot sweat rate was lower when wearing shoes and did not increase with exercise intensity which may be indicative of hidromeiosis. Despite this, sweat distribution patterns were similar between conditions and intensities. Foot discomfort and wetness perception were not dominated by regions of high sweat production but rather seemed to be dominated by tactile cues caused by foot movement in the shoe. Skin temperature did not seem to play a role in the perception of foot wetness.

\section{Application}

Foot regions identified as areas of high sweat production should be targeted in sock and shoe design, increasing ventilation and/or changing the shoe materials to aid moisture removal. Permeable running shoes have been associated with higher thermal comfort in comparison to impermeable running shoes during exercise (West et al. 2019). However, while targeting areas of high sweat production in shoe design is relevant for overall improvements in comfort, the results show that for local sensations the tactile component may be more important than the local wetness. This makes the tactile interaction between the foot, sock and shoe a second important area for development. A new approach for shoe designers would therefore be to target areas of low sweat production, high wetness perception (toes and heel) in terms of reducing the tactile afferents/cues generated between the foot, sock and shoe. This work will be integrated into a design 'toolbox' for use by sport scientists, engineers and designers, highlighting the considerations for the development of comfortable footwear.

Acknowledgements

The authors would like to thank those who participated in this experiment.

\section{Declaration of interests}

The research presented was funded by the adidas FUTURE team, Germany and the Environmental Ergonomics Research Centre, Loughborough Design School, Loughborough University. The authors were fully responsible for the conduct of the trial and the data. The authors declare that they have no conflict of interest. 
References

American College of Sports Medicine. 2017. ACSM's Guidelines for Exercise Testing and Prescription. 10th Edition.

Arezes, P. M., M. M. Neves, S. F. Teixeira, C. P. Leao, and J. L. Cunha. 2013. "Testing Thermal Comfort of Trekking Boots: An Objective and Subjective Evaluation." Applied Ergonomics 44 (4). Elsevier Ltd: 557-65. doi:10.1016/j.apergo.2012.11.007.

Auger, P, G Marguis, J Joly, and A Attye. 1993. "Epidemiology of Tinea Pedis in Marathon Runners: Prevalence of Occult Athlete's Foot.” Mycoses 36 ((1-2)): 3541.

Bergmann Tiest, Wouter M, N Dolfine Kosters, Astrid M L Kappers, Hein a M Daanen, Publisher Taylor, Wouter M Bergmann Tiest, and A M Hein. 2012. "Phase Change Materials and the Perception of Wetness." Ergonomics 55 (4): 508-12. doi:10.1080/00140139.2011.645886.

Candas, V, J P Libert, and J J Vogt. 1980. "Effect of Hidromeiosis on Sweat Drippage during Acclimation to Humid Heat.” European Journal of Applied Physiology and Occupational Phsyiology 44 (2): 123-33.

Dugan, Sheila A, and Krishna P Bhat. 2005. "Biomechanics and Analysis of Running Gait." Physical Medicine and Rehabilitation Clinics of North America 16: 603-21. doi:10.1016/j.pmr.2005.02.007.

Ferres, Helen M. 1960. "The Effect of Pressure on Sweating.” The Journal of Physiology 151: 591-97.

Filingeri, D., D. Fournet, S. Hodder, and G. Havenith. 2014. "Why Wet Feels Wet? A Neurophysiological Model of Human Cutaneous Wetness Sensitivity.” Journal of Neurophysiology 112 (6): 1457-69. doi:10.1152/jn.00120.2014.

Filingeri, Davide, and George Havenith. 2015. "Human Skin Wetness Perception: Psychophysical and Neurophysiological Bases.” Temperature 2 (1): 86-104. doi:10.1080/23328940.2015.1008878.

Fogarty, AL, R Barlett, V Ventenat, and G Havenith. 2007. "Regional Foot Sweat Rates during a 65-Minute Uphill Walk with a Backpack." In Proceedings of the 12th 
International Conference on Environmental Ergonomics, 283-84.

Fukazawa, Takako, and George Havenith. 2009. "Differences in Comfort Perception in Relation to Local and Whole Body Skin Wettedness.” European Journal of Applied Physiology 106 (1): 15-24. doi:10.1007/s00421-009-0983-z.

Havenith, George, Alison Fogarty, Rebecca Bartlett, Caroline J. Smith, and Vincent Ventenat. 2008. "Male and Female Upper Body Sweat Distribution during Running Measured with Technical Absorbents.” European Journal of Applied Physiology 104 (2): 245-55. doi:10.1007/s00421-007-0636-z.

Hwang, K and Baik, SH. 1997. "Distribution of Hairs and Sweat Glands on the Bodies of Korean Adults: A Morphometric Study." Acta Anatomica 158: 112-20.

Irzmanska, E., J. K. Dutkiewicz, and R. Irzmanski. 2013. "New Approach to Assessing Comfort of Use of Protective Footwear with a Textile Liner and Its Impact on Foot Physiology." Textile Research Journal, 1-11. doi:10.1177/0040517513507362.

ISO15831. 2004. "Clothing - Physiological Effects - Measurement of Thermal Insulation by Means of a Thermal Manikin.”

ISO 10551. 2001. Ergonomics of the Thermal Environment-assessment of the Influence of the Thermal Environment Using Subjective Judgement Scales. Geneva: International Standard.

Kerslake, D McK. 1972. The Stress of Hot Environments. Vol. 29. CUP Archive. Kuklane, K, and I I Holmer. 1998. "Effect of Sweating on Insulation of Footwear." Int J Occup Saf Ergon 4 (2): 123-36. doi:10.1080/10803548.1998.11076385.

Kuno, Yas. 1956. Human Perspiration. Thomas.

Machado-Moreira, Christiano A., Joanne N. Caldwell, Igor B. Mekjavic, and Nigel A S Taylor. 2008. "Sweat Secretion from Palmar and Dorsal Surfaces of the Hands during Passive and Active Heating." Aviation Space and Environmental Medicine 79 (11): 1034-40. doi:10.3357/ASEM.2354.2008.

Machado-Moreira, Christiano A., Foske M. Smith, Anne M J van dem Heuvel, Igor B. Mekjavic, and Nigel A S Taylor. 2008. "Sweat Secretion from the Torso during Passively-Induced and Exercise-Related Hyperthermia." European Journal of Applied Physiology 104 (2): 265-70. doi:10.1007/s00421-007-0646-х. 
Morris, N B, M N Cramer, S G Hodder, G Havenith, and O Jay. 2013. “A Comparison between the Technical Absorbent and Ventilated Capsule Methods for Measuring Local Sweat Rate.” Journal of Applied Physiology 114 (6): 816-23. doi:10.1152/japplphysiol.01088.2012.

Nadel, Ethan R, Robert W Bullard, and J A J Stolwijk. 1971. "Importance of Skin Temperature in the Regulation of Sweating." Journal of Applied Physiology 31 (1): 80-87.

Raccuglia, Margherita, Kolby Pistak, Christian Heyde, Jianguo Qu, Ningtao Mao, Simon Hodder, and George Havenith. 2017. "Human Wetness Perception of Fabrics under Dynamic Skin Contact.” Textile Research Journal, 4051751771690. doi:10.1177/0040517517716905.

Raccuglia, Margherita, Benjamin Sales, Christian Heyde, George Havenith, and Simon Hodder. 2018. "Clothing Comfort during Physical Exercise - Determining the Critical Factors." Applied Ergonomics 73 (April). Elsevier: 33-41. doi:10.1016/j.apergo.2018.05.014.

Ramanathan, NL. 1964. “A New Weighting System for Mean Surface Temperature of the Human Body." Journal of Applied Physiology 19 (3): 531-33.

Shimazaki, Yasuhiro, and Masaaki Murata. 2015. "Effect of Gait on Formation of Thermal Environment inside Footwear.” Applied Ergonomics 49. Elsevier Ltd: 5562. doi:10.1016/j.apergo.2015.01.007.

Smith, Caroline J., and George Havenith. 2011. "Body Mapping of Sweating Patterns in Male Athletes in Mild Exercise-Induced Hyperthermia.” European Journal of Applied Physiology 111 (7): 1391-1404. doi:10.1007/s00421-010-1744-8.

—. 2012. "Body Mapping of Sweating Patterns in Athletes: A Sex Comparison." Medicine and Science in Sports and Exercise 44 (12): 2350-61. doi:10.1249/MSS.0b013e318267b0c4.

—. 2019. "Upper Body Sweat Mapping Provides Evidence of Relative Sweat Redistribution towards the Periphery Following Hot-Dry Heat Acclimation.” Temperature 0 (0). Taylor \& Francis: 1-16. doi:10.1080/23328940.2019.1570777. Smith, Caroline J., Christiano A. Machado-Moreira, Gijs Plant, Simon Hodder, George Havenith, and Nigel A.S. Taylor. 2013. "Design Data for Footwear: Sweating 
Distribution on the Human Foot." International Journal of Clothing Science and Technology 25 (1): 43-58. doi:10.1108/09556221311292200.

Sulzberger, M. B., T. A. Cortese, L. Fishman, and H. S. Wiley. 1966. "Studies on Blisters Produced by Friction. I. Results of Linear Rubbing and Twisting Technics." The Journal of Investigative Dermatology 47 (5): 456-65. doi:10.1038/jid.1966.169.

Szabo, G. 1967. "The Regional Anatomy of the Human Integument with Special Reference to the Distribution of Hair Follicles, Sweat Glands and Melanocytes." Philosophical Transactions of the Royal Society of London 252 (779): 447-85.

Taylor, Nigel A S, Joanne N. Caldwell, and Igor B. Mekjavic. 2006. "The Sweating Foot: Local Differences in Sweat Secretion during Exercise-Induced Hyperthermia.” Aviation Space and Environmental Medicine 77 (10): 1020-27.

Weiner, J S. 1945. “The Regional Distribution of Sweating.” The Journal of Physiology 104: $32-40$.

West, A.M., D. Schönfisch, A. Picard, J. Tarrier, S. Hodder, and G. Havenith. 2019. "Shoe Microclimate: An Objective Characterisation and Subjective Evaluation." Applied Ergonomics 78 (July 2018). Elsevier: 1-12. doi:10.1016/j.apergo.2019.01.010. 
Table 1 Descriptive data for all foot regions sampled during shod and nude experimental trials. Light grey shading indicates a significantly higher mean sweat rate for the comparison between Nude-I1 and Shod-I1. Dark grey shading indicates a significantly higher mean sweat rate for the comparison between Shod-I1 and Shod-I2. Level of significance: $* p<0.05, * * p<0.01, * * * p<0001$.

Figure 1 Location of absorbent pads to the right foot for the assessment of foot sweat distribution. Site codes: 1 - toe $1 ; 2$ - toe $2 ; 3$ - toe 3 ; 4- toe 4 ; 5- toe 5 ; 6- plantar ball; 7 plantar outer; 8- plantar arch; 9- plantar heel; 10-medial dorsal; 11- central dorsal; 12 lateral dorsal; 13- medial ankle; 14- lateral ankle.

Figure 2 (a) thermal sensation scale; (b) wetness perception scale; (c) stickiness scale; (d) thermal comfort scale and (e) reference sheet used to highlight foot regions for subjective evaluation.

Figure 3 Regional foot sweat distribution for trained female runners assessed during low intensity running $\left(55 \% \mathrm{VO}_{2 \max }\right)$ without shoes (nude) and during low and high intensity running (55\% and $75 \% \mathrm{VO}_{2 \max }$, respectively) with shoes (shod) in moderately warm conditions $\left(25^{\circ} \mathrm{C}, 50 \% \mathrm{RH}\right)$.

Figure 4 Foot (a) thermal sensation (b) wetness perception (c) stickiness and (d) thermal comfort for nude and shod experimental trials. * denotes significance from previous time point for all shoe conditions $(p<0.001)$. $\dagger$ denotes significance from nude $(p<0.05)$.

Figure 5 Perceptual votes for (a) thermal sensation, (b) wetness perception, (c) stickiness and (d) thermal comfort taken as the final vote prior to pad application for four foot regions during nude and shod experimental trials. \#significantly greater than sole *significantly greater than dorsal. 


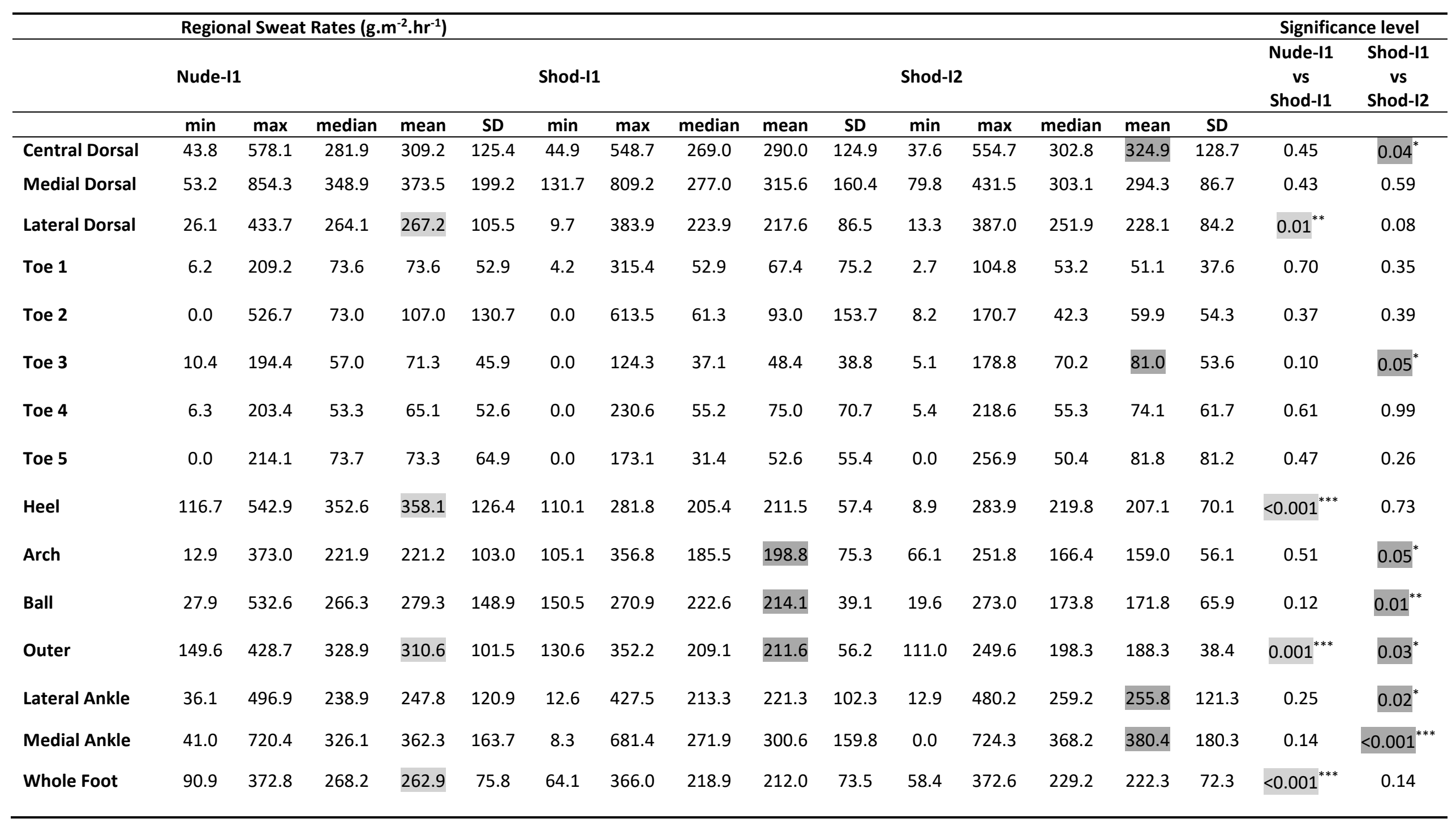



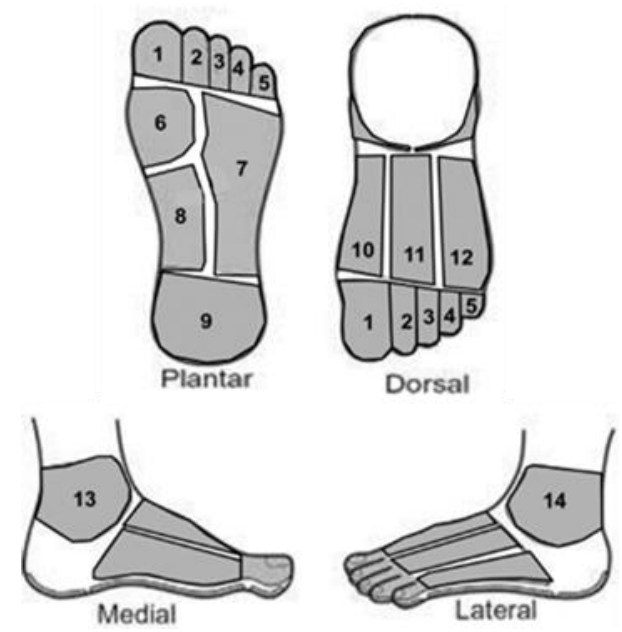
(a)

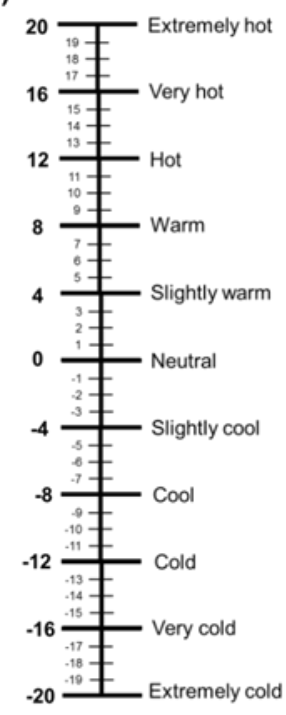

(b)

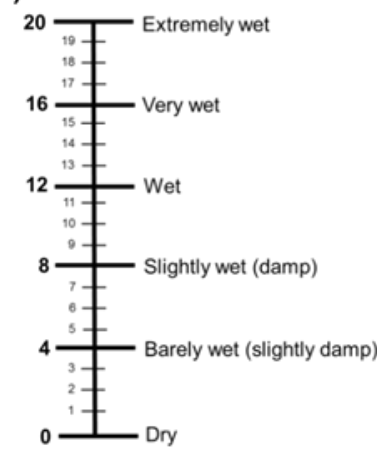

(c)

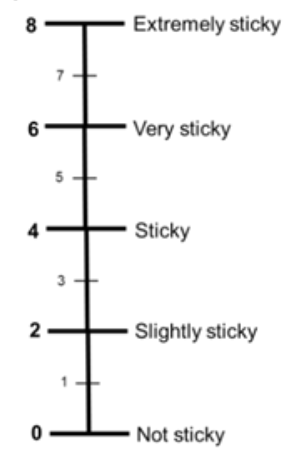

(d)

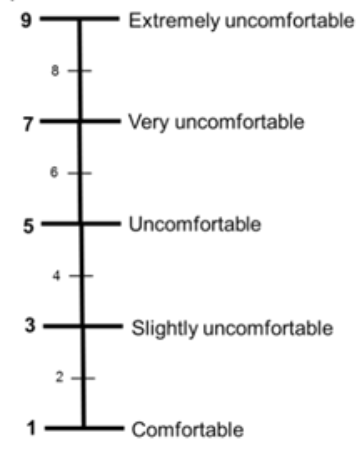

(e)

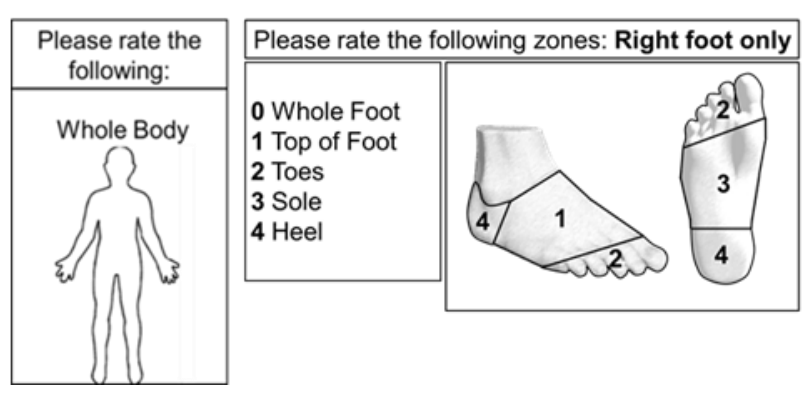


NUDE

LOW INTENSITY RUNNING

$\begin{array}{lll}74 \quad 107 & 71\end{array}$
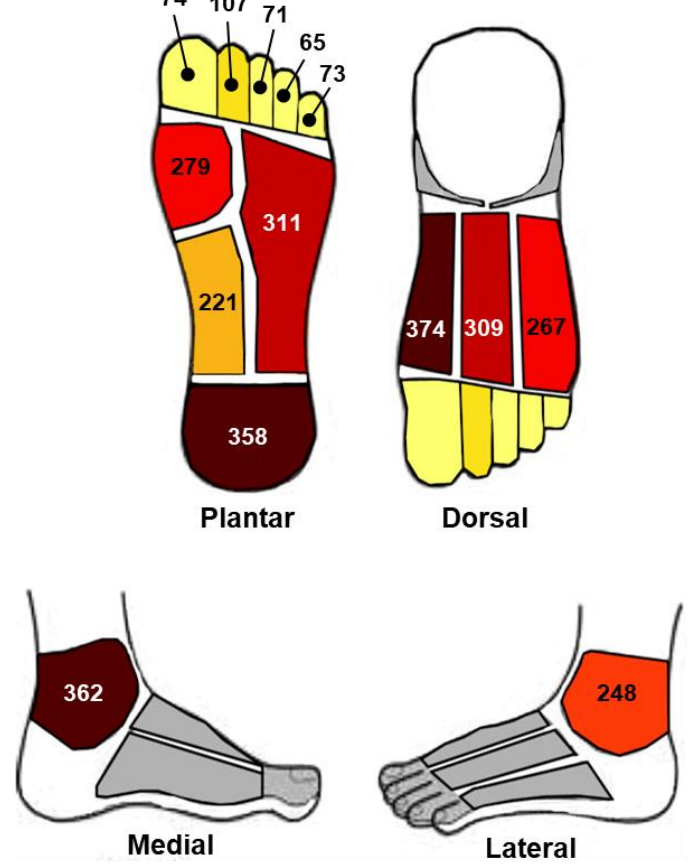

SHOD

LOW INTENSITY RUNNING

$\begin{array}{lll}67 & 93 & 48\end{array}$

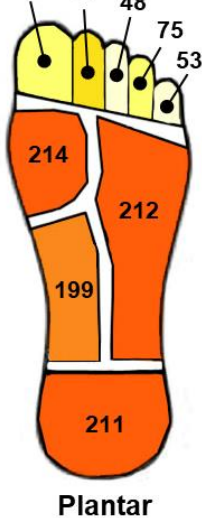

Plantar
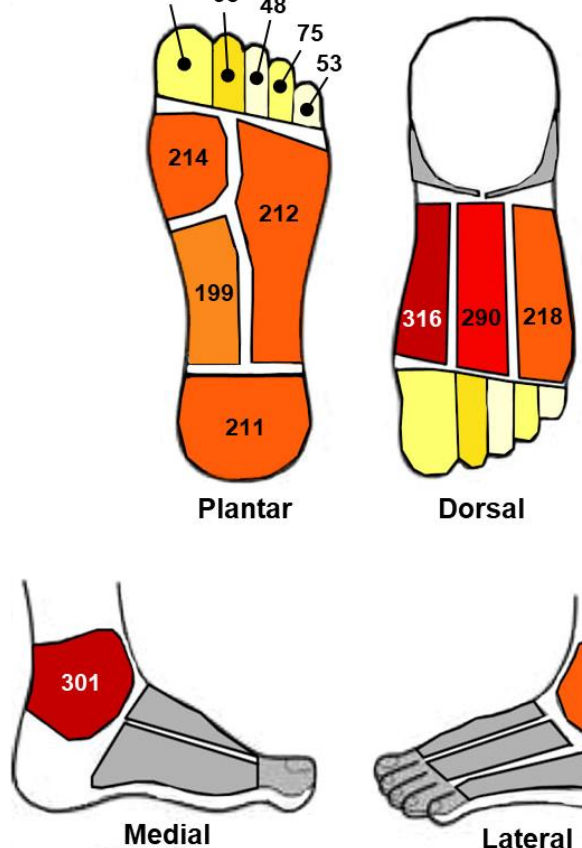

Medial

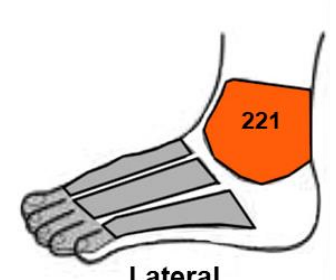

SHOD

HIGH INTENSITY RUNNING

$\begin{array}{lll}51 \quad 60 & 81\end{array}$
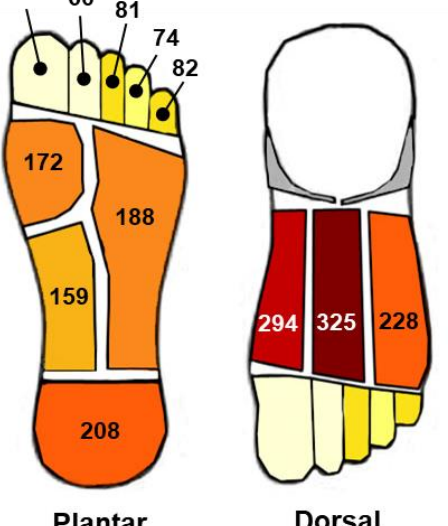

Sweat rate $\left(\mathrm{g} \cdot \mathrm{m}^{-2} \mathrm{~h}-1\right)$

$351-380$
$321-350$

291-320

261-290

231-260

201-230

171-200

$141-170$

111-140

$81-110$
$61-80$

$<60$ 


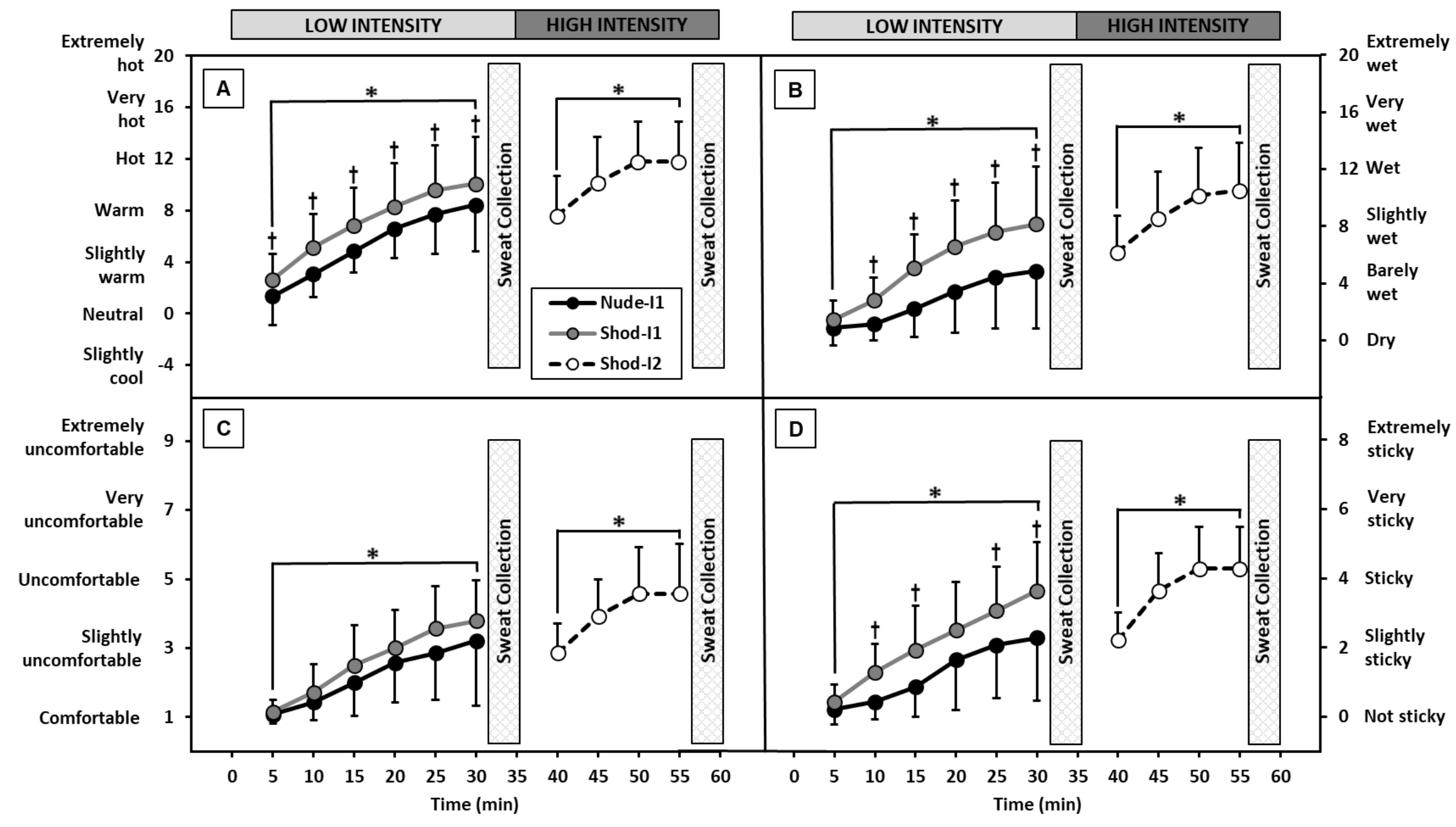




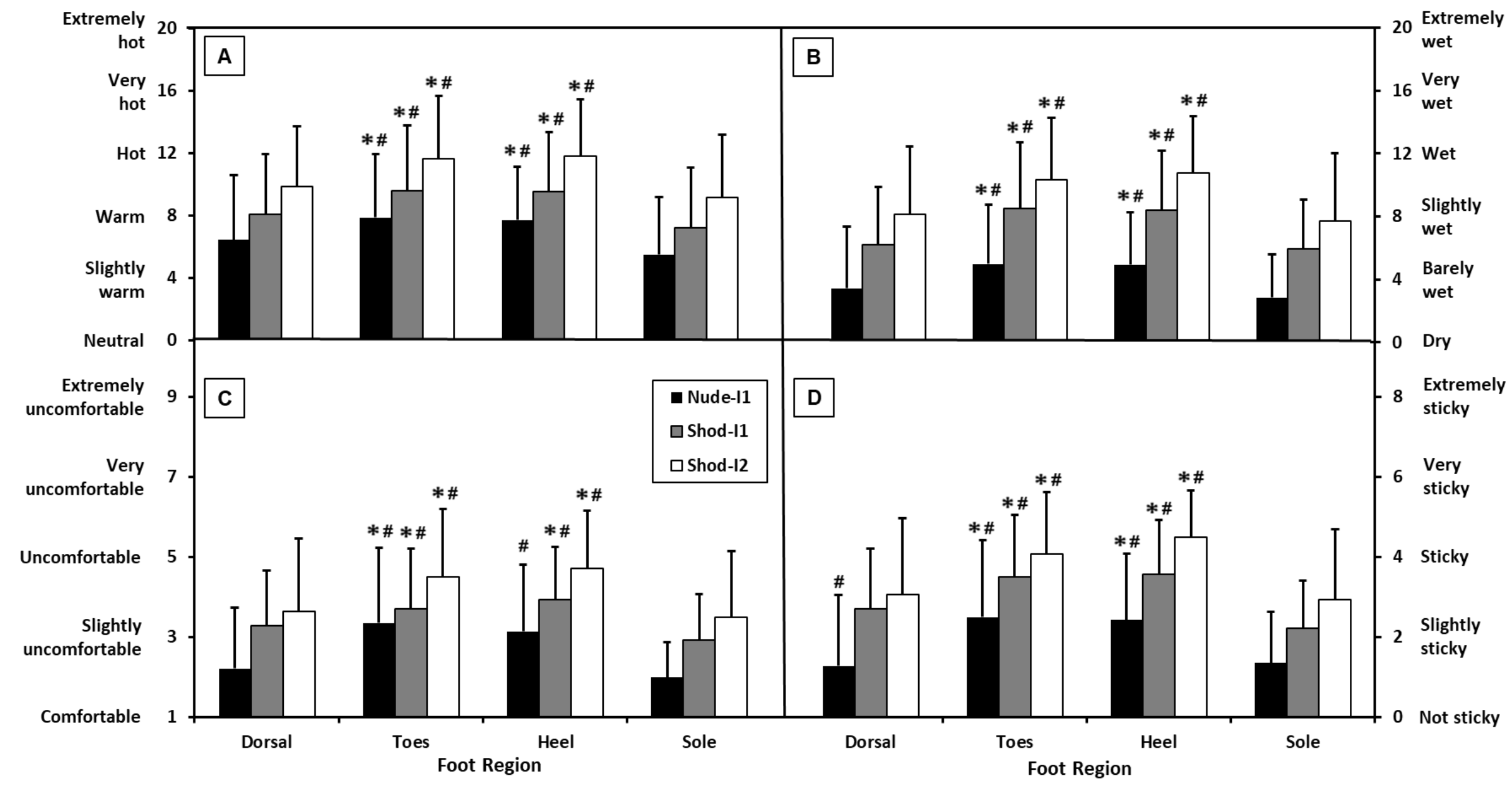

